\title{
Photonic RF Down-Converter Based on Optomechanical Oscillation
}

\author{
Mani Hossein-Zadeh, Member, IEEE, and Kerry J. Vahala, Member, IEEE
}

\begin{abstract}
We demonstrate all-optical radio-frequency (RF) down-conversion in a silica microtoroid optomechanical (OM) oscillator. Preliminary results show that the OM oscillator can simultaneously serve as mixer and local oscillator in a photonic homodyne RF-receiver architecture.
\end{abstract}

Index Terms-Down-conversion, optomechanical (OM) oscillator.

\section{INTRODUCTION}

$\mathbf{P}$ HOTONIC processing of radio-frequency (RF) signals using active and passive optical devices is an emerging technology with a wide range of applications in RF-over-fiber communication systems and photonic RF-receiver modules. Recently, the observation of self-sustained optomechanical (OM) oscillation in silica microtoroids [1], [2] has created new possibilities for designing RF-photonic devices. Although, through detailed experimental and theoretical studies, we have studied the fundamental properties of these oscillators [3], the concept of $\mathrm{OM}$ oscillation has not been employed in any RF-photonic device and its applications have been limited to a platform for studying fundamental physical phenomena. Here we propose a configuration where the $\mathrm{OM}$ oscillator can serve as an RF-frequency down-converter in the optical domain. Preliminary results show that an OM oscillator can simultaneously function as the nonlinear element and the local oscillator (LO) to down-convert the baseband signal from a standard suppressed-carrier RF signal. At this stage the OM down-converter may not be a feasible replacement for its electronic counterparts, however the fundamental idea lays the foundation for further research in this relatively young field.

\section{PhOTONIC HOMOdYNe RF DOWN-CONVERSION}

In an electronic homodyne receiver [Fig. 1(a)], the baseband signal is down-converted from the RF carrier by mixing the received signal with an LO that has the same frequency as the RF carrier $\left(f_{\mathrm{RF}}\right)$. The mixing occurs in an RF mixer through the nonlinearities of electronic elements (such as diodes and transistors). The LO also uses electronic components as well as

Manuscript received August 28, 2007; revised November 6, 2007. This work was supported in part by the Defense Advanced Research Projects Agency (DARPA), in part by Caltech Lee Center for Advanced Networking, and in part by Caltech Center for Physics of Information.

The authors are with the Applied Physics Department, California Institute of Technology, Pasadena, CA 91125 USA (e-mail: mhz@caltech.edu; vahala@caltech.edu).

Digital Object Identifier 10.1109/LPT.2007.912991
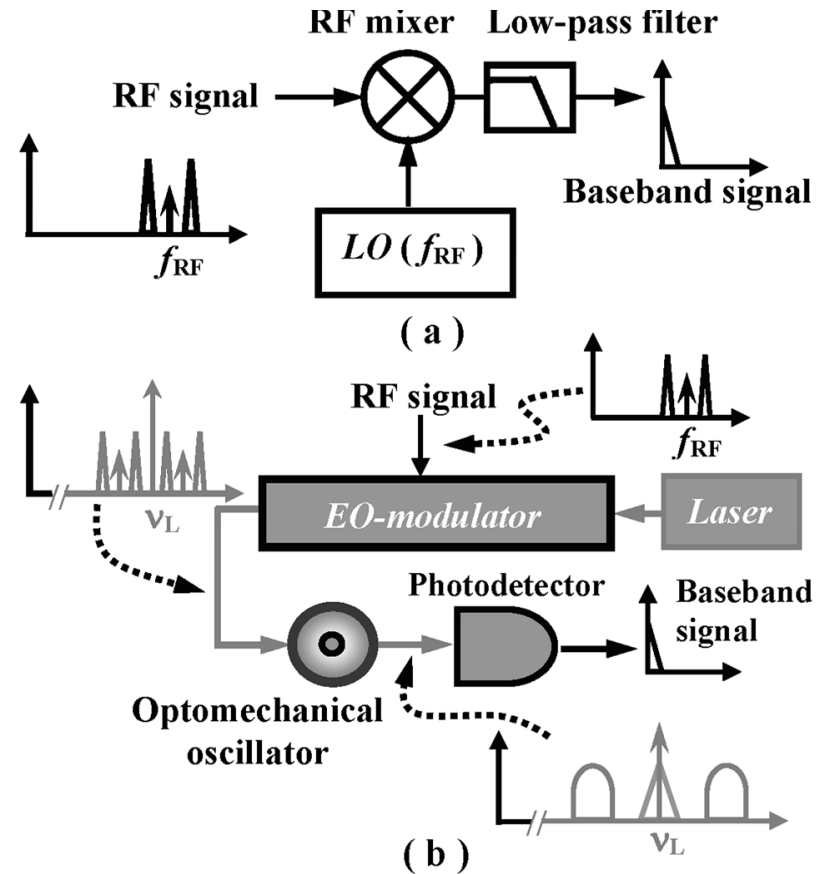

Fig. 1. (a) Schematic diagram of a standard electronic homodyne RF downconverter. (b) Schematic diagram of the photonic RF down-converter based on microtoroidal OM oscillator and the RF/optical spectrum of the signal while it flows through the system (indicated by dotted arrows).

an electromechanical reference resonator such as quartz crystal. In our proposed architecture [Fig. 1(b)], the received RF signal modulates the amplitude of an optical carrier $\left(\nu_{L} \approx 194 \mathrm{THz}\right)$ in an electrooptic modulator. The modulated optical wave is then fed to a microtoroidal silica resonator and finally the transmitted optical power is detected in a photodetector with a bandwidth matched to the baseband frequency.

When the optical input power $\left(P_{\text {in }}\right)$ to the microtoroid is larger than the threshold power for self-sustained OM oscillations [2], [3] and the OM oscillation frequency $\left(f_{\text {mech }}\right)$ is equal to the RF-carrier frequency $\left(f_{\mathrm{RF}}\right)$, the optical mixing inside the microtoroid generates a baseband component on the optical carrier, and therefore, upon detection, the photocurrent will be baseband-modulated (the high-frequency RF components are filtered out through the slow response of the photodetector). In other words, the OM oscillation combined with the nonlinear optical transfer function of the microtoroid (Lorentzian) reproduce the combined function of the LO and the mixer in an electronic down-converter [Fig. 1(a)]. Note that the bandwidth of the loaded optical resonance imposes an upper limit on the maximum frequency of the baseband signal. Fig. 2(a) shows the diagram of the experimental setup used for the proof-of-concept demonstration. 


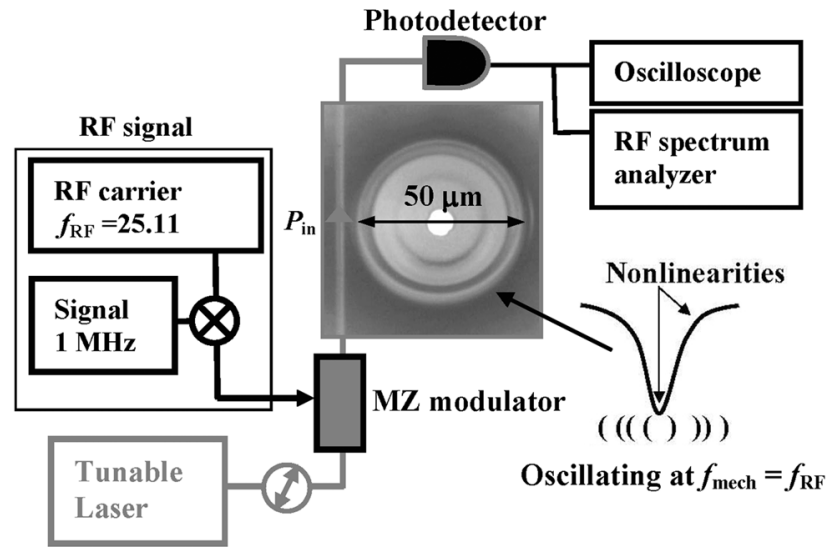

Fig. 2. Schematic diagram of the experimental setup. The chosen optical mode of the microtoroid has an intrinsic and loaded optical quality factor of $1 \times$ $10^{7}$ and $0.5 \times 10^{7}$, respectively. The mechanical mode has a frequency of $25.11 \mathrm{MHz}$ and quality factor of about $Q_{\text {mech }} \sim 4000$.

The RF signal is an RF carrier (25.11 MHz) modulated by a single-tone $(1 \mathrm{MHz})$ baseband signal (with suppressed carrier modulation format). This signal modulates the output of a tunable laser $(\lambda \sim 1550 \mathrm{~nm})$ in a Mach-Zehnder modulator. The modulated optical power is then coupled to a silica microtoroid resonator using a fiber-taper and the transmitted optical power is detected in a photodetector. An oscilloscope and an RF spectrum analyzer are used to control the optical detuning and analyzing the spectrum of the detected power.

The threshold optical input power for self-sustained OM oscillations $\left(P_{\mathrm{th}}\right)$ is $120 \mu \mathrm{W}$ and the mechanical mode has a frequency of $f_{\text {mech }}=25.11 \mathrm{MHz}$. Fig. 3 shows the RF spectrum of the transmitted optical power while the laser is tuned off (gray line) and on resonance (black line). The optical input power to the fiber-taper is $270 \mu \mathrm{W}$. If the laser wavelength is tuned off-resonance, the modulated optical wave is transmitted through the fiber-taper without being coupled to the microtoroid and the RF spectrum of the detected optical power is identical with the spectrum of a suppressed carrier modulated RF wave (the signal that is driving the MZ modulator). When the laser is tuned inside the optical resonance, the circulating optical power initiates the self-sustained OM oscillation. OM oscillation modulates the amplitude of the optical wave and boosts the modulated RF power at $25.11 \mathrm{MHz}$ [Fig. 3(a)]. Meanwhile since the laser is tuned to the nonlinear section of the optical transfer function, the oscillation mixes with the RF-sidebands $(25.11 \pm$ $1 \mathrm{MHz}$ ) and down converts the single tone baseband [Fig. 3(b)]. In this proof-of-concept experiment, the down-conversion efficiency (defined as the ratio of baseband optical power to the sum of optical powers at each RF-sideband) is about $15 \%$. Note that the efficiency of the OM RF-down-conversion is directly proportional to the optical input power, as well as the optical and mechanical quality factors. The phase noise of the downconverted signal can be dominated either by the $\mathrm{OM}$ oscillator phase noise or by the phase noise of the carrier frequency in the received RF signal depending on their relative magnitudes (the larger one dominates). Note that at room temperature the phase noise of the OM oscillator is limited by thermal noise in the microtoroid structure [3] and it scales inversely with mechanical quality factor $\left(Q_{\text {mech }}\right)$.
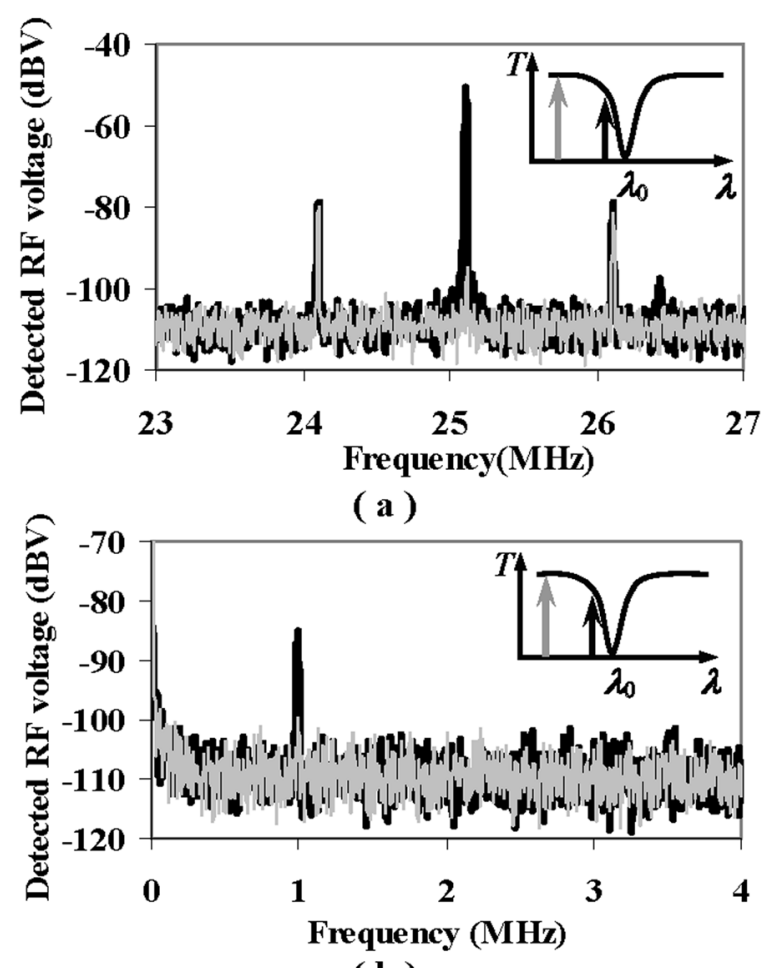

(b)

Fig. 3. Off-resonance (gray) and on-resonance (black) RF spectrum of the transmitted optical power (a) near $f_{\mathrm{RF}}$ and (b) near the baseband frequency. The insets show the relative detuning of the laser wavelength and the optical resonance for each case ( $T$ is the transmitted optical power).

\section{NONLINEARITY AND FREQUENCY SWICHING IN OM OSCILLATOR}

\section{A. Nonlinearity of the Oscillation}

The amplitude of self-sustained oscillation and the nonlinearity of the optical transfer function are the two key elements in the down-conversion process. For each microtoroid, the amplitude of $\mathrm{OM}$ oscillation is determined by the ratio $P_{\mathrm{in}} / P_{\mathrm{th}}$ and the laser detuning from resonance $\left(\Delta \nu=\nu_{L}-\nu_{0}\right)$. The detuning which maximizes the oscillation amplitude depends on intrinsic properties of the microtoroid as well as optical coupling [3]. The detuning also determines the segment of the Lorentzian response that is used for transmission and, therefore, controls the strength of the nonlinear behavior. For optimized down-conversion, it is desired to have the maximum OM gain at a detuning that also results in maximum nonlinear behavior. Experimental results show that typically the optimized detuning is about $60 \%-80 \%$ of the loaded linewidth of the optical resonance. Here we use the strength of the second-harmonic component in the spectrum of the detected optical power from the OM oscillator as a measure for the mixing efficiency (square-law mixer approximation). Fig. 4 shows the ratio of the detected power at the fundamental OM frequency $\left(P_{1}\right)$ and its secondharmonic $\left(P_{2}\right)$ plotted against $P_{\mathrm{in}} / P_{\mathrm{th}}$. The two sets of data correspond to two different microtoroids with different loaded optical- $Q \mathrm{~s}\left(Q_{L}\right)$ and mechanical frequencies $(f)$. The insets show the alignment of the laser frequency and the lower sidebands $\left(\nu_{L}, \nu_{L}-f, \nu_{L}-2 f\right)$ relative to the optical mode lineshape function for each case (note that the upper sidebands, $\nu_{L}+f$ and $\nu_{L}+2 f$, are filtered out by the transfer function). 


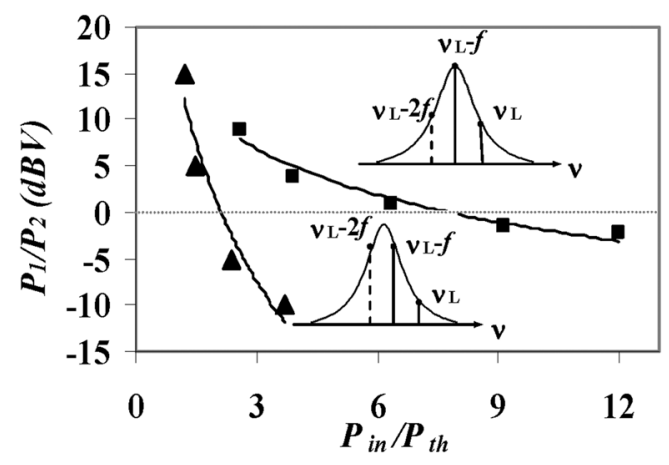

Fig. 4. Ratio of the detected power at fundamental OM frequency $(f)$ and its second-harmonic $(2 f)$ plotted against $P_{\mathrm{in}} / P_{\mathrm{th}}$. The two sets of data correspond to two different microtoroids. The insets show the relative alignment of the laser frequency $\left(\nu_{L}\right)$ and the sidebands generated by OM oscillation relative to the optical lineshape function. For each microtoroid, the laser detuning from resonance $(\Delta \nu)$ is optimized for maximum value of $\left(P_{1} / P_{2}\right)$.

The laser detuning from resonance $\left(\Delta \nu=\nu_{0}-\nu_{L}\right)$ is optimized for a maximum value of $\left(P_{1} / P_{2}\right)$. As we expected, increasing $P_{\text {in }} / P_{\text {th }}$ drives the oscillator to the nonlinear regime. The difference between these two cases can be explained by the fact that the relative amplitude of the OM sidebands are modified through the Lorentzian response of the cavity (Fig. 4, insets).

So for the first case (rectangles), the second-harmonic is suppressed by the low-frequency tail of the Lorentzian, while for the second case (triangles), the fundamental frequency and the second-harmonic coexist within the optical resonance. This measurement is a proof for the possibility of strong oscillation in the nonlinear regime and hence efficient mixing. It also shows that for each microtoroid there are optimal values of $P_{\mathrm{in}} / P_{\mathrm{th}}$ and $Q_{L}$ that should be used for OM RF mixing process.

\section{B. Frequency Switching}

Usually at a given optical input power and optical coupling strength, the microtoroid oscillates at one of its eigen mechanical modes. However, by tailoring the geometry of the microtoroid and adjusting the loaded- $Q$, the OM oscillation frequency can be switched by changing the wavelength detuning from resonance. To demonstrate this possibility, we have chosen a different microtoroid with fundamental and third mechanical eigen frequencies of 35 and $91 \mathrm{MHz}$. Fig. 5(a) shows the transmitted optical power as the laser wavelength is slowly swept (10 sweeps/s) through the resonant wavelength. The triangular shape of the transmission (as opposed to a Lorentzian) is an artifact of the thermooptical resonant shift in conjunction with the slow scan rate. The dark broadened regions in the wavelength scan correspond to optical detunings at which the OM gain overcomes the mechanical loss and the ensuing regenerative OM oscillation modulates the optical power. As shown in Fig. 5(a), the OM oscillation occurs at two distinct wavelength detunings. RF spectrum analysis of these oscillations shows that they correspond to two mechanical modes of the microtoroid. Fig. 5(b) shows the RF spectrum of the detected optical output power while the laser wavelength is tuned to two different wavelengths indicated by the arrows on Fig. 5(a). The black and gray

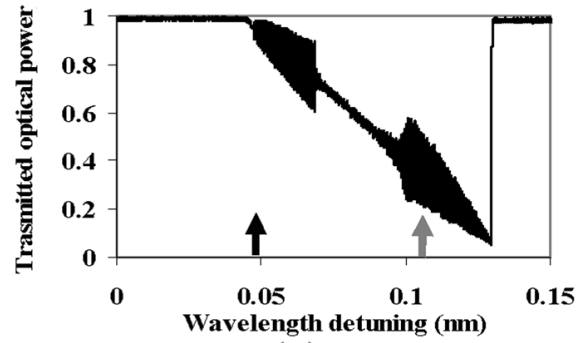

(a)

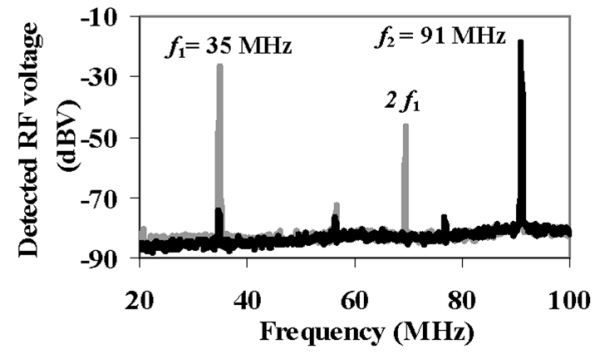

(b)

Fig. 5. (a) Normalized transmission spectrum near a high- $Q$ optical mode. The dark broadened regions correspond to OM oscillation at 91 and $35 \mathrm{MHz}$. The loaded- $Q$ of the chosen resonance is $1.5 \times 10^{6}$. (b) RF spectrum of the detected optical output power.

arrows correspond to the black and gray traces in Fig. 5(b). This degree of freedom could be useful in the context of the OM RF down-converter since it enables data down-conversion from two distinct career frequencies only by tuning the laser wavelength within the optical resonance.

\section{CONCLUSION}

We have demonstrated a photonic RF down-conversion technique based on self-sustained OM oscillations in a silica microtoroid. Note that previously a self-homodyne RF photonic receiver was demonstrated based on nonlinear modulation in an electrooptic microdisk [4]. However, in that architecture, the LO is eliminated by using the transmitted carrier modulation format and as a result it suffers from low power efficiency as well as incompatibility with standard RF transceivers for long-distance communication. In contrast, the dual functionality of the OM oscillator (oscillator/mixer) makes it compatible with suppressed carrier modulation format.

\section{REFERENCES}

[1] D. K. Armani, T. J. Kippenberg, S. M. Spillane, and K. J. Vahala, "Ultra-high-Q toroid microcavity on a chip," Nature, vol. 421, pp. 925-929, 2003.

[2] T. J. Kippenberg, H. Rokhsari, T. Carmon, A. Scherer, and K. J. Vahala, "Analysis of radiation-pressure induced mechanical oscillation of an optical microcavity," Phys. Rev. A, vol. 95, p. 033901, 2005.

[3] M. Hossein-Zadeh, H. Rokhsari, A. Hajimiri, and K. J. Vahala, "Characterization of a radiation-pressure-driven micromechanical oscillator," Phys. Rev. A, vol. 74, p. 023813, 2006.

[4] M. Hossein-Zadeh and A. F. J. Levi, " $14.6 \mathrm{GHz} \mathrm{LiNbO}_{3}$ microdisk photonic self-homodyne RF receiver," IEEE Trans. Microw. Theory Tech., vol. 54, no. 2, pt. 2, pp. 821-831, Feb. 2006. 